\title{
Impact of a multidisciplinary diabetic foot clinic on patient outcomes
}

Phillips LA, BMedSci, MBChB Hons, CT1 Doctor.

Osborne L, MBChB, MRCP, Consultant Diabetologist.

Department of Diabetes and Endocrinology, Victoria Hospital, Kirkcaldy, Fife.

\section{Background}

Foot disease in diabetes is associated with significant morbidity and mortality ${ }^{1}$. Diabetic foot disease requires complex care with input from a range of healthcare professionals. Scottish Intercollegiate Guidelines Network guideline 116 recommends that all patients with diabetes and foot ulceration are seen at a multidisciplinary foot clinic $^{2}$.

\section{Aims}

Our aim was to assess the impact of the trial introduction of a multidisciplinary foot clinic on patient outcomes within one NHS health board (NHS Fife.)

\section{Methods}

The inclusion criteria for this study was a diagnosis of diabetes mellitus, with at least one foot ulcer which had not healed within 28 days of presentation. Retrospective data was collected regarding patient demographics, wound healing, amputation rates, re-ulceration rates, cardiovascular risk profile, and mortality, for patients attending the multidisciplinary foot clinic between September 2012 and September 2013. This was compared with data collected from patients receiving specialist podiatrist care only, between September 2011 and March 2012.

\section{Results}

Data was collected from 31 multidisciplinary clinic patients and 137 patients from the specialist podiatry clinic.

1. Wound healing: Our data demonstrates a trend towards a higher rate of wound healing amongst patients attending the multidisciplinary clinic $(72.5 \%$ vs $62.1 \%$, $p=0.2)$.

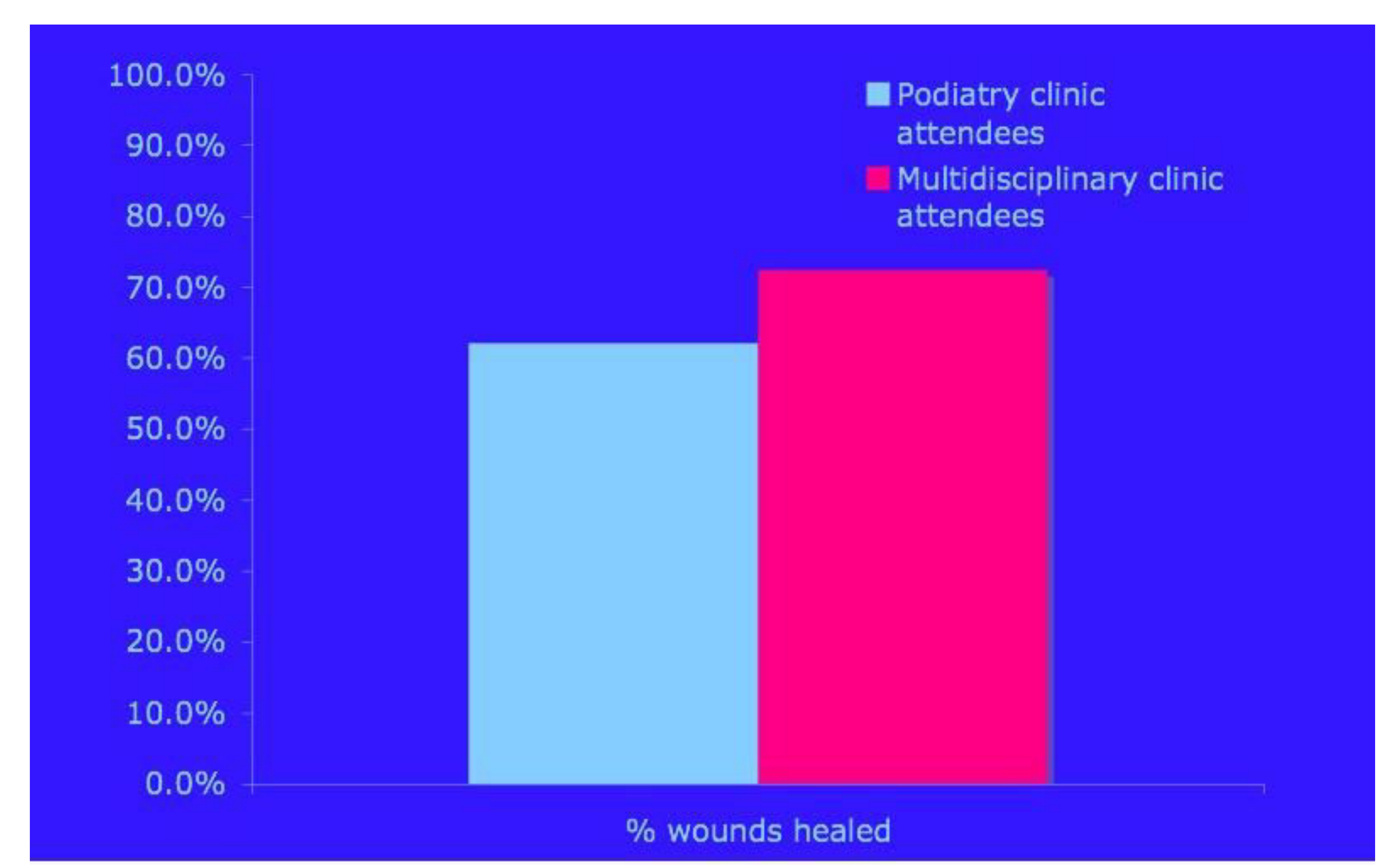

2. Amputations: There was a trend towards a lower rate of amputations amongst multidisciplinary clinic attendees (15\% vs $17.6 \%, p=0.69$.) There was a significant reduction in the need for major amputations amongst multidisciplinary attendees ( $2.5 \%$ vs $11 \%, p=0.04$.)

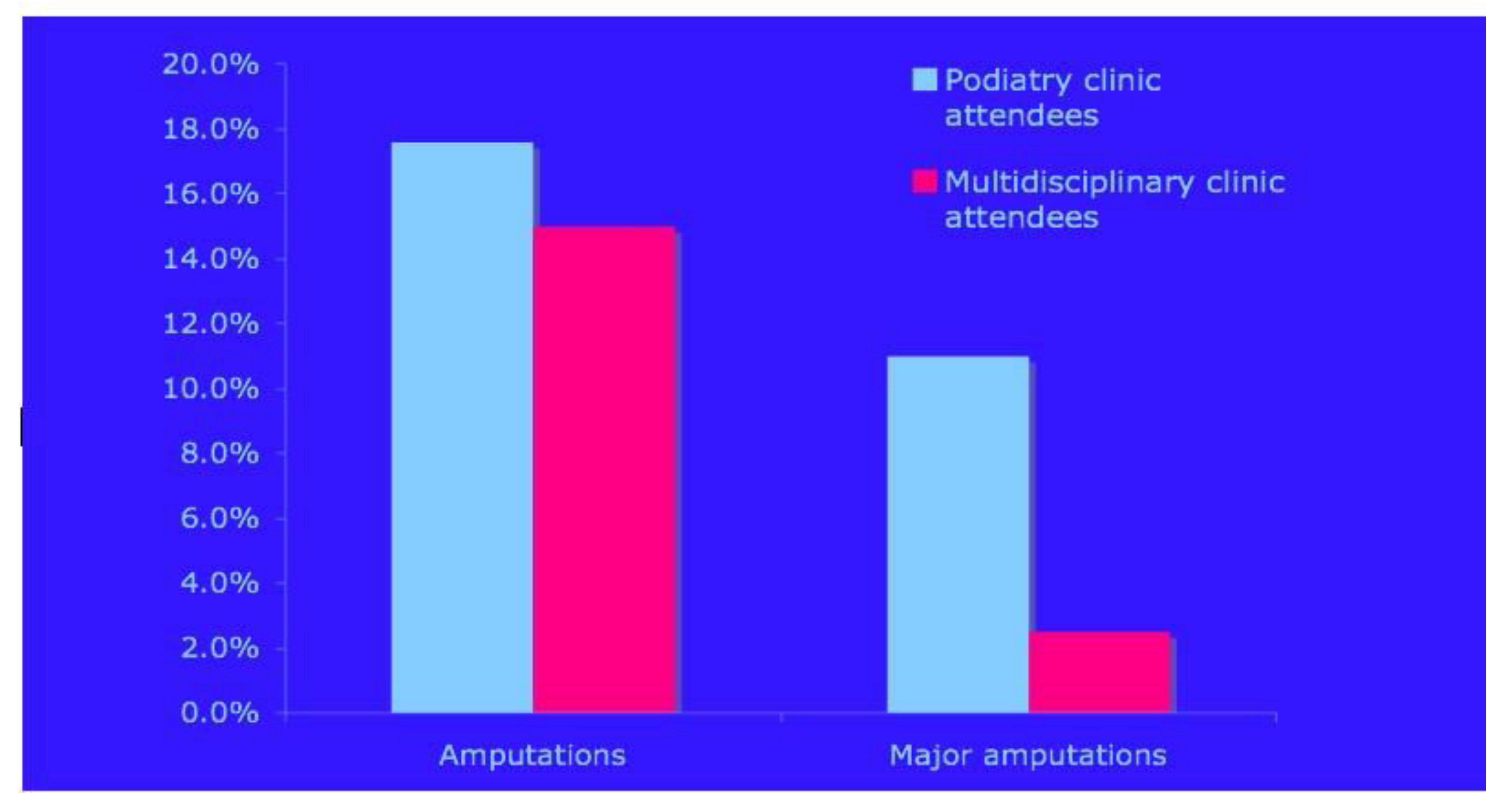

3. Wound healing post amputation: Following amputation, surgical wound healing time was shorter for multidisciplinary foot clinic attendees compared with podiatry clinic attendees (207.5 days vs 304.4 days, $p=0.52$ ).

4. Re-ulceration: Re-ulceration at 6 months was similar for both cohorts (25.8\% vs $28.5 \%, p=0.77$.)

\section{Cardiovascular risk profile:}

Glycaemic control: There was a significant improvement in $\mathrm{HbA}_{1} \mathrm{C}$ amongst multidisciplinary clinic attendees $(79.5 \mathrm{mmol} / \mathrm{mol}$ to $65.5 \mathrm{mmol} / \mathrm{mol}, \mathrm{p}=0.00002)$. No significant improvement in glycaemic control was seen amongst podiatry clinic attendees $(p=0.29)$.

Blood pressure: Amongst multidisciplinary clinic attendees the mean systolic blood pressure reduced from $136 \mathrm{mmHg}$ to $127 \mathrm{mmHg},(p=0.026)$, and the mean diastolic blood pressure reduced from $74 \mathrm{mmHg}$ to $69 \mathrm{mmHg},(p=0.006)$. No significant improvement was seen amongst podiatry clinic attendees $(p=0.6$ and 0.17 for systolic and diastolic blood pressures respectively).

Cholesterol: There was a significant reduction in mean serum cholesterol amongst multidisciplinary clinic attendees $(4.28 \mathrm{mmol} / \mathrm{l}$ to $3.67 \mathrm{mmol} / \mathrm{l}, \mathrm{p}=0.0009)$. No improvement in lipid profile was seen amongst podiatry clinic attendees $(p=0.53)$.

6. Mortality: Mortality at 12 months was significantly reduced amongst multidisciplinary clinic attendees compared with podiatry clinic attendees (0\% vs $19 \%$, $\mathrm{p}=0.01$ ).

\section{Conclusions}

Our study demonstrates that the introduction of a multidisciplinary foot clinic has had a positive impact on patient outcomes. We aim to secure resources to establish an ongoing multidisciplinary diabetic foot clinic in NHS Fife.

\section{REFERENCES}

1.Fox CS, Coady S, Sorlie PD, Levy D, Meigs JB, D'Agnostio RB, Wilson PW, Savage PJ. Trends in cardiovascular complications of diabetes. JAMA. 2004:292;2495-2499

2.Scottish Intercollegiate Guidelines Network (SIGN) Guidelines 116. Management of diabetes: Edinburgh, UK, 2010. Available from URL: http://www.sign.ac.uk/pdf/sign116.pdf. 